(C)2008 IEEE. Personal use of this material is permitted. However, permission to reprint/republish this material for advertising or promotional purposes or for creating new collective works for resale or redistribution to servers or lists, or to reuse any copyrighted component of this work in other works must be obtained from the IEEE. 


\title{
ROBUST LINEAR RECEIVER DESIGN FOR MULTI-ACCESS SPACE-TIME BLOCK CODED MIMO SYSTEMS USING STOCHASTIC OPTIMIZATION
}

\author{
Yue Rong Sergiy A. Vorobyov Alex B. Gershman \\ Communication Systems Group, Darmstadt University of Technology \\ Merckstr. 25, 64283 Darmstadt, Germany
}

\begin{abstract}
Traditional receiver algorithms developed for multiple-input multiple-output (MIMO) wireless systems are based on the assumption that the channel state information (CSI) is precisely known at the receiver. However, in real environments the exact CSI may be unavailable. In this paper, we address the problem of robustness of multi-access spacetime block coded (STBC) MIMO systems against imperfect CSI. We propose a class of linear receivers which guarantee the robustness against CSI errors with a certain selected probability. The proposed formulations of robust receivers are given by probability-constrained optimization problems which can be simplified to convex forms. The latter convex problems can be efficiently solved using standard optimization methods.
\end{abstract}

\section{INTRODUCTION}

In uplink cellular communications with multiple receive antennas at the base station and transmit antennas at each mobile station, spatial diversity techniques can be employed to increase the capacity [1] and improve the immunity to fading [2], [3]. Among numerous space-time codes developed up to date, orthogonal space-time block codes (OSTBCs) [2], [3] are particularly attractive for practical applications because they enable a very simple (linear) maximum likelihood (ML) decoding in the point-to-point communication case and, at the same time, achieve the full diversity order.

Unfortunately, in the multi-access MIMO case, the optimal ML receiver becomes prohibitively expensive and, hence, suboptimal linear multiuser receivers have gained recently much interest as computationally attractive alternatives [4], [5]. However, most of known multi-access MIMO receivers assume the exact knowledge of the channel state information (CSI) of at least the user of interest. When the exact CSI is unavailable, the performance of these receivers may degrade severely.

Motivated by the latter fact, the problem of robust linear multiuser MIMO receiver design has been recently addressed in [6], where the worst-case optimization approach [7] has been used to improve the robustness of the minimum variance (MV) receivers of [5] against the CSI mismatches. However, the worst-case approach appears to be overly pessimistic and, therefore, it may lead to unnecessary performance degradation.

In this paper, we propose a class of robust linear multiuser MIMO receivers which are less conservative than the worst-case receivers of [6]. They guarantee the robustness against CSI errors with a certain selected probability. The earlier (high-complexity) versions of such receivers have been reported in [8]. The mathematical formulation of the receiver design problem is given in terms of probabilityconstrained stochastic optimization problem (the so-called chance programming) [9]. We prove the convexity of the corresponding stochastic optimization problems and develop techniques to solve them, based on the assumption that the CSI mismatch is Gaussian. They convert the original stochastic optimization problems into the second-order cone programming (SOCP) problems which can be efficiently solved using modern convex optimization methods [10].

\section{BACKGROUND}

Let us consider an uplink multiuser MIMO communication system. The transmitters are assumed to have the same number of antennas and to encode information-bearing symbols using the same OSTBC ${ }^{1}$. The received signal is given by

$$
\boldsymbol{Y}=\sum_{i=1}^{I} \boldsymbol{X}_{i} \boldsymbol{H}_{i}+\boldsymbol{V}
$$

where

$$
\begin{aligned}
\boldsymbol{Y} & \triangleq\left[\begin{array}{lll}
\boldsymbol{y}^{T}(1) & \cdots & \boldsymbol{y}^{T}(T)
\end{array}\right]^{T} \\
\boldsymbol{X}_{i} & \triangleq\left[\begin{array}{llll}
\boldsymbol{x}_{i}^{T}(1) & \cdots & \boldsymbol{x}_{i}^{T}(T)
\end{array}\right]^{T} \\
\boldsymbol{V} & \triangleq\left[\begin{array}{llll}
\boldsymbol{v}^{T}(1) & \cdots & \boldsymbol{v}^{T}(T)
\end{array}\right]^{T}
\end{aligned}
$$

are the matrices of the received signals, transmitted signals of the $i$ th transmitter, and white Gaussian noise, respectively; $\boldsymbol{H}_{i}$ is the $N \times M$ complex channel matrix between

\footnotetext{
${ }^{1}$ These assumptions are only needed for notational simplicity and can be relaxed [5].
} 
the $i$ th transmitter and the receiver; $I$ is the number of transmitters; $T$ is the block length; and $(\cdot)^{T}$ denotes the transpose. Here

$$
\begin{aligned}
\boldsymbol{y}(t) & \triangleq\left[y_{1}(t) \cdots y_{M}(t)\right] \\
\boldsymbol{x}_{i}(t) & \triangleq\left[x_{i, 1}(t) \cdots x_{i, N}(t)\right] \\
\boldsymbol{v}(t) & \triangleq\left[v_{1}(t) \cdots v_{M}(t)\right]
\end{aligned}
$$

are the complex row vectors of the received signals, transmitted signals of the $i$ th transmitter, and noise, respectively.

We denote the complex information-bearing symbols of the $i$ th transmitter prior to space-time encoding as $\boldsymbol{s}_{i} \triangleq$ $\left[s_{i, 1} \cdots s_{i, K}\right]^{T}$. It can be readily verified that the matrix $\boldsymbol{X}\left(s_{i}\right)$ can be written as [11], [12]

$$
\boldsymbol{X}\left(\boldsymbol{s}_{i}\right)=\sum_{k=1}^{K}\left(\boldsymbol{C}_{k} \operatorname{Re}\left\{s_{i, k}\right\}+\boldsymbol{D}_{k} \operatorname{Im}\left\{s_{i, k}\right\}\right)
$$

where $\boldsymbol{C}_{k} \triangleq \boldsymbol{X}\left(\tilde{\boldsymbol{e}}_{k}\right), \boldsymbol{D}_{k} \triangleq \boldsymbol{X}\left(j \tilde{\boldsymbol{e}}_{k}\right), j=\sqrt{-1}$ and $\tilde{\boldsymbol{e}}_{k}$ is the $K \times 1$ vector having one in the $k$ th position and zeros elsewhere. Using (2), one can rewrite (1) as [5], [12]

$$
\underline{\boldsymbol{Y}}=\sum_{i=1}^{I} \boldsymbol{A}\left(\boldsymbol{H}_{i}\right) \underline{\boldsymbol{s}_{i}}+\underline{\boldsymbol{V}}
$$

where the "underline" operator for any matrix $\boldsymbol{P}$ is defined as

$$
\underline{\boldsymbol{P}}=\left[\begin{array}{l}
\operatorname{vec}(\operatorname{Re}\{\boldsymbol{P}\}) \\
\operatorname{vec}(\operatorname{Im}\{\boldsymbol{P}\})
\end{array}\right]
$$

$\operatorname{vec}(\cdot)$ is the vectorization operator stacking all columns of a matrix on top of each other, and the $2 M T \times 2 K$ real matrix $\boldsymbol{A}\left(\boldsymbol{H}_{i}\right)$ is defined as [5], [12]

$$
\begin{aligned}
\boldsymbol{A}\left(\boldsymbol{H}_{i}\right) & \triangleq\left[\boldsymbol{C}_{1} \boldsymbol{H}_{i} \cdots \underline{\boldsymbol{C}_{K} \boldsymbol{H}_{i}} \underline{\boldsymbol{D}_{1} \boldsymbol{H}_{i}} \cdots \underline{\boldsymbol{D}_{K} \boldsymbol{H}_{i}}\right] \\
& \triangleq\left[\boldsymbol{a}_{1}\left(\boldsymbol{H}_{i}\right) \cdots \boldsymbol{a}_{2 K}\left(\boldsymbol{H}_{i}\right)\right]
\end{aligned}
$$

Without any loss of generality, we can assume that the first user is the desired one. The estimate of the data vector $\underline{\hat{s}}_{1}$ at the output of a linear receiver can be expressed as [5]

$$
\underline{\hat{\boldsymbol{s}}_{1}}=\boldsymbol{W}^{T} \underline{\boldsymbol{Y}}
$$

where $\boldsymbol{W}=\left[\boldsymbol{w}_{1} \cdots \boldsymbol{w}_{2 K}\right]$ is the $2 M T \times 2 K$ real matrix of the receiver weight coefficients, and $\boldsymbol{w}_{k}$ is the $2 M T \times 1$ real weight vector that corresponds to decoding the $k$ th entry of $\underline{s_{1}}$. The problem is to find the matrix $\boldsymbol{W}$ that separates the signals from different users.

The similarity of the vectorized multiple-access MIMO model (3) and models used in array processing gives an opportunity to design the matrix $W$ using the MV principle. In particular, in [5] it has been proposed to estimate each entry of $\underline{s_{1}}$ by minimizing the receiver output power while preserving a unity gain for this particular entry of $\underline{s_{1}}$, that is,

$$
\min _{\boldsymbol{w}_{k}} \boldsymbol{w}_{k}^{T} \hat{\boldsymbol{R}} \boldsymbol{w}_{k} \quad \text { subject to } \quad \boldsymbol{a}_{k}^{T}\left(\boldsymbol{H}_{1}\right) \boldsymbol{w}_{k}=1
$$

for all $k=1, \ldots, 2 K$, where

$$
\hat{\boldsymbol{R}}=\frac{1}{J} \sum_{i=1}^{J} \underline{\boldsymbol{Y}_{i}}{\underline{\boldsymbol{Y}_{i}}}^{T}
$$

is the sample estimate of the $2 M T \times 2 M T$ full rank covariance matrix $\boldsymbol{R} \triangleq \mathrm{E}\left\{\underline{\boldsymbol{Y}} \underline{\boldsymbol{Y}}^{T}\right\}$ of the vectorized data, $\boldsymbol{Y}_{i}$ is the $i$ th received data block, and $\mathrm{E}\{\cdot\}$ denotes the statistical expectation.

The solution to (5) is given by [5]

$$
\boldsymbol{w}_{k}=\frac{1}{\boldsymbol{a}_{k}^{T}\left(\boldsymbol{H}_{1}\right) \hat{\boldsymbol{R}}^{-1} \boldsymbol{a}_{k}\left(\boldsymbol{H}_{1}\right)} \hat{\boldsymbol{R}}^{-1} \boldsymbol{a}_{k}\left(\boldsymbol{H}_{1}\right)
$$

for $k=1, \ldots, 2 K$.

To incorporate the self-interference cancellation feature into (5), it has been proposed in [5] to use additional zeroforcing constraints $\boldsymbol{w}_{k}^{T} \boldsymbol{a}_{l}\left(\boldsymbol{H}_{1}\right)=0$ for all $l \neq k$. These constraints guarantee that self-interference is completely rejected. With such additional constraints, the problem (5) can be reformulated as

$$
\min _{\boldsymbol{W}} \operatorname{tr}\left\{\boldsymbol{W}^{T} \hat{\boldsymbol{R}} \boldsymbol{W}\right\} \quad \text { subject to } \quad \boldsymbol{A}^{T}\left(\boldsymbol{H}_{1}\right) \boldsymbol{W}=\boldsymbol{I}_{2 K}
$$

where $\operatorname{tr}\{\cdot\}$ denotes the trace of a matrix. The solution to (7) can be written in the form of the following MV receiver [5]

$$
\boldsymbol{W}_{\mathrm{MV}}=\hat{\boldsymbol{R}}^{-1} \boldsymbol{A}\left(\boldsymbol{H}_{1}\right)\left(\boldsymbol{A}^{T}\left(\boldsymbol{H}_{1}\right) \hat{\boldsymbol{R}}^{-1} \boldsymbol{A}\left(\boldsymbol{H}_{1}\right)\right)^{-1} .
$$

To improve the performance of (5) and (7) in case of small sample size, it has been additionally proposed in [5] to apply ad hoc diagonal loading, i.e., to use matrix $\hat{\boldsymbol{R}}+$ $\nu \boldsymbol{I}_{2 M T}$ instead of $\hat{\boldsymbol{R}}$ in (6) and (8), where $\nu$ is the diagonal loading factor.

It can be seen from (5) and (7) that the MV receivers require the CSI of the user of interest. However, in practice, it is unrealistic to obtain the exact CSI at the receiver. Therefore, the performance of the MV receivers may be subject to a severe degradation due to CSI imperfections. To improve the robustness of these receivers against CSI errors, modifications of (5) and (7) based on worst-case performance optimization have been proposed in [6], [13]. However, the worst-case approach may be overly pessimistic because the probability of occurrence of the worst-case mismatch may be quite low. Therefore, it may lead to unnecessary performance degradation. In this paper, we develop another, less conservative approach to robust linear MIMO receivers design which is based on stochastic programming [9]. 


\section{ROBUST LINEAR RECEIVERS BASED ON STOCHASTIC PROGRAMMING}

Let us consider the CSI error matrix $\boldsymbol{\Delta}_{i} \triangleq \boldsymbol{H}_{i}-\hat{\boldsymbol{H}}_{i}$ where $\boldsymbol{H}_{i}$ and $\hat{\boldsymbol{H}}_{i}$ denote the true channel matrix of the $i$ th user and its estimate, respectively. Using the notations of model (3), we can write

$$
\begin{aligned}
\boldsymbol{e}_{k}\left(\boldsymbol{\Delta}_{i}\right) & \triangleq \boldsymbol{a}_{k}\left(\boldsymbol{H}_{i}\right)-\boldsymbol{a}_{k}\left(\hat{\boldsymbol{H}}_{i}\right)=\underline{\boldsymbol{F}_{k} \boldsymbol{H}_{i}}-\underline{\boldsymbol{F}_{k} \hat{\boldsymbol{H}}_{i}} \\
& =\underline{\boldsymbol{F}_{k} \boldsymbol{\Delta}_{i}}
\end{aligned}
$$

for all $k=1, \ldots, 2 K$, where

$$
\boldsymbol{F}_{k}=\left\{\begin{array}{cl}
\boldsymbol{C}_{k}, & k=1, \ldots, K \\
\boldsymbol{D}_{k-K}, & k=K+1, \ldots, 2 K .
\end{array}\right.
$$

Note, that the last equality in (9) follows from the linearity of underline operator (4).

Let us obtain the receiver coefficient vector $\boldsymbol{w}_{k}$ for the $k$ th entry of $s_{1}$ as the solution of the following probabilityconstrained optimization problem

$$
\begin{array}{ll}
\min _{\boldsymbol{w}_{k}} & \boldsymbol{w}_{k}^{T} \hat{\boldsymbol{R}} \boldsymbol{w}_{k} \\
\text { s.t. } & \operatorname{Pr}\left\{\boldsymbol{w}_{k}^{T}\left(\boldsymbol{a}_{k}\left(\hat{\boldsymbol{H}}_{1}\right)+\boldsymbol{e}_{k}\left(\boldsymbol{\Delta}_{1}\right)\right) \geq 1\right\} \geq p
\end{array}
$$

where $p$ is a certain probability value which should be selected according to the quality of service $(\mathrm{QoS})$ requirements, and $\operatorname{Pr}\{\cdot\}$ denotes the probability operator whose form is assumed to be known. It is important to note that the probability bound $p$ in the problem (10)-(11) can be selected from the interval $(0,1)$ and it determines the amount of channel mismatch that is allowed at the receiver.

In the formulation (10)-(11), the receiver output power is minimized, while the distortionless response for $k$ th entry of $s_{1}$ is kept with a certain probability. Problem (10)-(11) is called in the optimization literature the chance-constrained or probability-constrained stochastic programming problem [9]. The constraint (11) can be also called non-outage probability constraint.

THEOREM 1: If $\left[\boldsymbol{\Delta}_{i}\right]_{n, m} \sim \mathcal{C N}\left(0, \sigma_{h}^{2}\right)$, where $\mathcal{C N}(\cdot, \cdot)$ stands for complex Gaussian distribution, and $p \in(0.5,1)$, then the optimization problem (10)-(11) is convex.

PROOF: The objective function (10) is a quadratic function of $\boldsymbol{w}_{k}$, and $\hat{\boldsymbol{R}}$ is positive definite. Therefore, (10) is convex.

Now we prove that the constraint (11) is also convex under the assumptions of the theorem. First, we note that $\boldsymbol{e}_{k}\left(\boldsymbol{\Delta}_{i}\right)$ depends linearly on $\boldsymbol{\Delta}_{i}$. Indeed, applying the underline operator (4) to (9) and using well-known properties of Kronecker product (denoted hereafter as $\otimes$ ) and $\operatorname{vec}(\cdot)$ operation, we can write that

$$
\begin{aligned}
& \boldsymbol{e}_{k}\left(\boldsymbol{\Delta}_{i}\right)=\left[\begin{array}{l}
\operatorname{vec}\left(\operatorname{Re}\left\{\boldsymbol{F}_{k} \boldsymbol{\Delta}_{i}\right\}\right) \\
\operatorname{vec}\left(\operatorname{Im}\left\{\boldsymbol{F}_{k} \boldsymbol{\Delta}_{i}\right\}\right)
\end{array}\right] \\
= & {\left[\begin{array}{cc}
\operatorname{Re}\left\{\boldsymbol{I}_{M} \otimes \boldsymbol{F}_{k}\right\} & -\operatorname{Im}\left\{\boldsymbol{I}_{M} \otimes \boldsymbol{F}_{k}\right\} \\
\operatorname{Im}\left\{\boldsymbol{I}_{M} \otimes \boldsymbol{F}_{k}\right\} & \operatorname{Re}\left\{\boldsymbol{I}_{M} \otimes \boldsymbol{F}_{k}\right\}
\end{array}\right]\left[\begin{array}{c}
\operatorname{vec}\left(\operatorname{Re}\left\{\boldsymbol{\Delta}_{i}\right\}\right) \\
\operatorname{vec}\left(\operatorname{Im}\left\{\boldsymbol{\Delta}_{i}\right\}\right)
\end{array}\right] . }
\end{aligned}
$$

Thus, (12) explicitly shows that $\boldsymbol{e}_{k}\left(\boldsymbol{\Delta}_{i}\right)$ is a linear combination of the real and imaginary parts of the elements of the channel mismatch matrix $\boldsymbol{\Delta}_{i}$. If the elements of $\boldsymbol{\Delta}_{i}$ are uncorrelated and have circular complex Gaussian distribution

$$
\left[\boldsymbol{\Delta}_{i}\right]_{n, m} \sim \mathcal{C N}\left(0, \sigma_{h}^{2}\right), \quad n=1, \ldots N, \quad m=1, \ldots, M
$$

then, using (12), we find that $\boldsymbol{e}_{k}\left(\boldsymbol{\Delta}_{i}\right)$ has multivariate real Gaussian distribution

$$
\boldsymbol{e}_{k}\left(\boldsymbol{\Delta}_{i}\right) \sim \mathcal{N}\left(\mathbf{0}_{2 M T}, \frac{\sigma_{h}^{2}}{2}\left(\boldsymbol{I}_{2 M} \otimes \boldsymbol{G}_{k} \boldsymbol{G}_{k}^{T}\right)\right)
$$

where $\mathcal{N}(\cdot, \cdot)$ stands for real Gaussian distribution, and

$$
\boldsymbol{G}_{k}=\left\{\begin{array}{cl}
\boldsymbol{C}_{k}, & k=1, \ldots, K \\
\operatorname{Im}\left\{\boldsymbol{D}_{k-K}\right\}, & k=K+1, \ldots, 2 K .
\end{array}\right.
$$

Since only $\boldsymbol{e}_{k}\left(\boldsymbol{\Delta}_{i}\right)$ is a random variable in the product $\boldsymbol{w}_{k}^{T}\left(\boldsymbol{a}_{k}\left(\hat{\boldsymbol{H}}_{i}\right)+\boldsymbol{e}_{k}\left(\boldsymbol{\Delta}_{i}\right)\right)$, and both $\boldsymbol{w}_{k}$ and $\boldsymbol{a}_{k}\left(\hat{\boldsymbol{H}}_{i}\right)$ are deterministic values, the random variable $\boldsymbol{w}_{k}^{T}\left(\boldsymbol{a}_{k}\left(\hat{\boldsymbol{H}}_{i}\right)+\right.$ $\left.\boldsymbol{e}_{k}\left(\boldsymbol{\Delta}_{i}\right)\right)$ has real Gaussian distribution

$$
\begin{aligned}
& \boldsymbol{w}_{k}^{T}\left(\boldsymbol{a}_{k}\left(\hat{\boldsymbol{H}}_{i}\right)+\boldsymbol{e}_{k}\left(\boldsymbol{\Delta}_{i}\right)\right) \sim \\
& \mathcal{N}\left(\boldsymbol{w}_{k}^{T} \boldsymbol{a}_{k}\left(\hat{\boldsymbol{H}}_{i}\right), \frac{\sigma_{h}^{2}}{2}\left\|\left(\boldsymbol{I}_{2 M} \otimes \boldsymbol{G}_{k}^{T}\right) \boldsymbol{w}_{k}\right\|^{2}\right)
\end{aligned}
$$

where $\|\cdot\|$ denotes the Euclidian norm of a vector.

Using the standard error function for Gaussian distribution

$$
\operatorname{erf}(x)=\frac{2}{\sqrt{\pi}} \int_{0}^{x} e^{-t^{2}} d t
$$

the left hand side of the constraint (11) can be written as

$$
\begin{aligned}
& \operatorname{Pr}\left\{\boldsymbol{w}_{k}^{T}\left(\boldsymbol{a}_{k}\left(\hat{\boldsymbol{H}}_{1}\right)+\boldsymbol{e}_{k}\left(\boldsymbol{\Delta}_{1}\right)\right) \geq 1\right\}= \\
& \frac{1}{2}-\frac{1}{2} \operatorname{erf}\left(\frac{1-\boldsymbol{w}_{k}^{T} \boldsymbol{a}_{k}\left(\hat{\boldsymbol{H}}_{1}\right)}{\sigma_{h}\left\|\left(\boldsymbol{I}_{2 M} \otimes \boldsymbol{G}_{k}^{T}\right) \boldsymbol{w}_{k}\right\|}\right) .
\end{aligned}
$$

Substituting (15) into (11), after some straightforward manipulations, we obtain the following constraint

$$
\operatorname{erf}\left(\frac{\boldsymbol{w}_{k}^{T} \boldsymbol{a}_{k}\left(\hat{\boldsymbol{H}}_{1}\right)-1}{\sigma_{h}\left\|\left(\boldsymbol{I}_{2 M} \otimes \boldsymbol{G}_{k}^{T}\right) \boldsymbol{w}_{k}\right\|}\right) \geq 2 p-1 .
$$

If $p>0.5$, then (16) is convex and can be written as

$$
\sigma_{h}\left\|\left(\boldsymbol{I}_{2 M} \otimes \boldsymbol{G}_{k}^{T}\right) \boldsymbol{w}_{k}\right\| \leq \frac{\boldsymbol{w}_{k}^{T} \boldsymbol{a}_{k}\left(\hat{\boldsymbol{H}}_{1}\right)-1}{\operatorname{erf}^{-1}(2 p-1)}
$$

where $\operatorname{erf}^{-1}(\cdot)$ denotes the inverse error function. The constraint (17) is called second-order cone (SOC) constraint.

Summarizing, both the objective function and the constraint are convex. Therefore, the problem (10)-(11) is convex. 
Although the problem (10)-(11) is convex, it is nonlinear and does not have closed-form solution. However, it can be equivalently converted to the following SOCP problem

$$
\begin{aligned}
\min _{\boldsymbol{w}_{k}, \chi} & \chi \\
\text { s.t. } & \left\|\boldsymbol{Z} \boldsymbol{w}_{k}\right\| \leq \chi \\
& \sigma_{h}\left\|\left(\boldsymbol{I}_{2 M} \otimes \boldsymbol{G}_{k}^{T}\right) \boldsymbol{w}_{k}\right\| \leq \frac{\boldsymbol{w}_{k}^{T} \boldsymbol{a}_{k}\left(\hat{\boldsymbol{H}}_{1}\right)-1}{\operatorname{erf}^{-1}(2 p-1)}
\end{aligned}
$$

where

$$
\hat{\boldsymbol{R}}=\boldsymbol{Z}^{T} \boldsymbol{Z}
$$

is the Cholesky factorization of $\hat{\boldsymbol{R}}$.

Using the standard optimization tools [15], the problem (18)-(20) can be solved with the complexity order of $\mathcal{O}\left(M^{3} T^{3}\right)[14]$.

It is interesting to note that for the Alamouti code [2], the constraint (20) is equivalent to

$$
\operatorname{erf}^{-1}(2 p-1) \sigma_{h}\left\|\boldsymbol{w}_{k}\right\| \leq \boldsymbol{w}_{k}^{T} \boldsymbol{a}_{k}\left(\hat{\boldsymbol{H}}_{1}\right)-1
$$

The expression (22) follows directly from the fact that for the Alamouti code $\boldsymbol{G}_{k} \boldsymbol{G}_{k}^{T}=\boldsymbol{I}_{2}$.

It is important to establish a connection between the receiver (18)-(20) for the case of the Alamouti code and the receiver developed in [6] based on the worst-case approach The latter receiver can be represented as the following optimization problem

$$
\begin{array}{cl}
\min _{\boldsymbol{w}_{k}, \chi} & \chi \\
\text { s.t. } & \left\|\boldsymbol{Z} \boldsymbol{w}_{k}\right\| \leq \chi \\
& \varepsilon\left\|\boldsymbol{w}_{k}\right\| \leq \boldsymbol{w}_{k}^{T} \boldsymbol{a}_{k}\left(\hat{\boldsymbol{H}}_{1}\right)-1
\end{array}
$$

where $\varepsilon$ is a constant which bounds the uncertainty region of the CSI mismatch, i.e., $\left\|\Delta_{1}\right\| \leq \varepsilon$. The problem (23)-(25) is equivalent to the problem (18)-(20) if (20) is substituted by (22) and if

$$
\varepsilon=\operatorname{erf}^{-1}(2 p-1) \sigma_{h} .
$$

Equation (26) shows the link between the probability $p$ (which is used in the proposed approach) and the parameter $\varepsilon$ of the worst-case-based receivers design in the case of Alamouti code.

In the problem (10)-(11), the self-interference and multiaccess interference are suppressed by minimizing the objective function (10). However, the guaranteed self-interference suppression is very important for the performance of multiuser MIMO receivers. See the discussion in [5]. Motivated by this, we propose to suppress self-interference with a certain selected probability, similar to the way how the distortionless response is maintained in the problem (10)(11). With such additional constraints, the new problem can be written as

$$
\begin{aligned}
\min _{\boldsymbol{w}_{k}, \boldsymbol{\delta}} & \boldsymbol{w}_{k}^{T} \hat{\boldsymbol{R}} \boldsymbol{w}_{k}+\|\boldsymbol{\delta}\|^{2} \\
\text { s.t. } & \operatorname{Pr}\left\{\boldsymbol{w}_{k}^{T}\left(\boldsymbol{a}_{k}\left(\hat{\boldsymbol{H}}_{1}\right)+\boldsymbol{e}_{k}\left(\boldsymbol{\Delta}_{1}\right)\right) \geq 1\right\} \geq p, \\
& \operatorname{Pr}\left\{\sigma_{1}\left|\boldsymbol{w}_{k}^{T}\left(\boldsymbol{a}_{l}\left(\hat{\boldsymbol{H}}_{1}\right)+\boldsymbol{e}_{l}\left(\boldsymbol{\Delta}_{1}\right)\right)\right| \leq \delta_{l}\right\} \geq p, \\
& l=1, \ldots, 2 K, \quad l \neq k
\end{aligned}
$$

where $\boldsymbol{\delta}=\left[\delta_{1}, \ldots, \delta_{k-1}, \delta_{k+1}, \cdots, \delta_{2 K}\right]^{T}$ is the $(2 K-$ 1) $\times 1$ vector whose values limit the contribution of selfinterference, and $\sigma_{1}$ is the standard deviation of the waveform of the desired user.

THEOREM 2: If $\left[\boldsymbol{\Delta}_{i}\right]_{n, m} \sim \mathcal{C N}\left(0, \sigma_{h}^{2}\right)$ and $p \in(0.5,1)$, then the optimization problem (27)-(29) is convex.

PROOF: The objective function (27) is obviously convex. It has been proven earlier in this paper that the constraint (28) is convex if $\left[\boldsymbol{\Delta}_{i}\right]_{n, m} \sim \mathcal{C N}\left(0, \sigma_{h}^{2}\right)$ and $p \in$ $(0.5,1)$. Moreover, it has been proven in [8] that the constraints (29) are also convex if $\left[\boldsymbol{\Delta}_{i}\right]_{n, m} \sim \mathcal{C N}\left(0, \sigma_{h}^{2}\right)$ and $p \in(0.5,1)$. Therefore, the problem (27)-(29) is convex. $\square$

The problem (27)-(29) can be simplified to SOCP problem [10], [14] in the following way. Let us use the Cholesky factorization of $\hat{\boldsymbol{R}}(21)$ and introduce a new vector

$$
\zeta \triangleq\left[\left(\boldsymbol{Z} \boldsymbol{w}_{k}\right)^{T}, \boldsymbol{\delta}^{T}\right]^{T}
$$

Then, minimizing $\|\boldsymbol{\zeta}\|$ is equivalent to minimizing (27).

The deterministic equivalent form of the constraint (28) is given by (17), which is a SOC constraint. However, the constraints (29) can not be equivalently converted to SOC constraints. To enable such conversion, we apply the Chebyshev inequality to the constraint (29). The latter inequality states that for any random variable $\xi$ and any positive real number $\alpha$,

$$
\mathrm{P}\{|\xi| \geq \alpha\} \leq \frac{\mathrm{E}\left\{\xi^{2}\right\}}{\alpha^{2}} .
$$

Since the constraints (29) share the same structure, we further discuss only the $l$ th constraint pointing that the same manipulations can be applied to other constraints. Under the assumption that $\boldsymbol{e}_{l}\left(\boldsymbol{\Delta}_{1}\right)$ has Gaussian distribution, we have

$$
\mathrm{E}\left\{\left|\boldsymbol{w}_{k}^{T}\left(\boldsymbol{a}_{l}\left(\hat{\boldsymbol{H}}_{1}\right)+\boldsymbol{e}_{l}\left(\boldsymbol{\Delta}_{1}\right)\right)\right|^{2}\right\}=\boldsymbol{w}_{k}^{T} \boldsymbol{Q}_{l}\left(\hat{\boldsymbol{H}}_{1}\right) \boldsymbol{w}_{k}
$$

where

$$
\boldsymbol{Q}_{l}\left(\hat{\boldsymbol{H}}_{1}\right) \triangleq \boldsymbol{a}_{l}\left(\hat{\boldsymbol{H}}_{1}\right) \boldsymbol{a}_{l}^{T}\left(\hat{\boldsymbol{H}}_{1}\right)+\frac{\sigma_{h}^{2}}{2}\left(\boldsymbol{I}_{2 M} \otimes \boldsymbol{G}_{l} \boldsymbol{G}_{l}^{T}\right) .
$$
obtain

Applying (30) and (31) to the $l$ th constraint of (29), we

$$
\sigma_{1}^{2} \boldsymbol{w}_{k}^{T} \boldsymbol{Q}_{l}\left(\hat{\boldsymbol{H}}_{1}\right) \boldsymbol{w}_{k} \leq(1-p) \delta_{l}^{2}
$$

Finally, the constraint (32) can be converted into the following SOC constraint

$$
\sigma_{1}\left\|\boldsymbol{T}_{l}^{T}\left(\hat{\boldsymbol{H}}_{1}\right) \boldsymbol{w}_{k}\right\| \leq \sqrt{(1-p)} \delta_{l}
$$




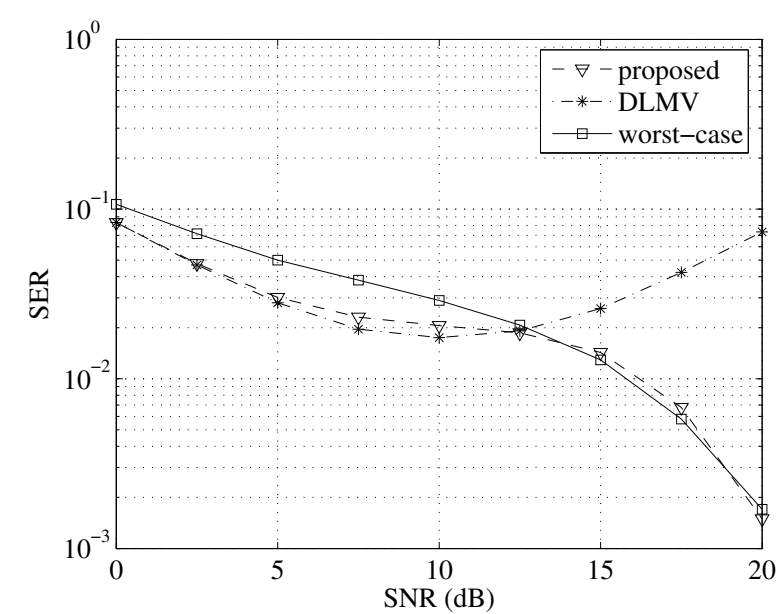

Fig. 1. SER versus SNR; First example.

where $\boldsymbol{T}_{l}\left(\hat{\boldsymbol{H}}_{1}\right) \boldsymbol{T}_{l}^{T}\left(\hat{\boldsymbol{H}}_{1}\right)=\boldsymbol{Q}_{l}\left(\hat{\boldsymbol{H}}_{1}\right)$. Then, the optimization problem (27)-(29) can be relaxed to the following SOCP problem

$$
\begin{aligned}
\min _{\boldsymbol{w}_{k}, \boldsymbol{\delta}, \tau} & \tau \\
\text { s.t. } & \|\boldsymbol{\zeta}\| \leq \tau \\
& \sigma_{h}\left\|\left(\boldsymbol{I}_{2 M} \otimes \boldsymbol{G}_{k}^{T}\right) \boldsymbol{w}_{k}\right\| \leq \frac{\boldsymbol{w}_{k}^{T} \boldsymbol{a}_{k}\left(\hat{\boldsymbol{H}}_{1}\right)-1}{\mathrm{erf}^{-1}(2 p-1)} \\
& \sigma_{1}\left\|\boldsymbol{T}_{l}^{T}\left(\hat{\boldsymbol{H}}_{1}\right) \boldsymbol{w}_{k}\right\| \leq \sqrt{(1-p)} \delta_{l} \\
& l=1, \ldots, 2 K, \quad l \neq k .
\end{aligned}
$$

Note that the relaxed problem (33)-(36) can be easily solved using standard and highly efficient interior-point methods, for example, using SeDuMi package [15]. Using the primal-dual potential reduction method, the problem (33)(36) can be solved with the complexity order $\mathcal{O}\left(M^{3} T^{3} K^{3}\right)$ [14].

\section{SIMULATIONS}

An uplink cellular communication system with multiple transmitters is simulated in this section. Throughout the simulations, we assume a single receiver equipped with $M=8$ antennas. The number of transmitters varies in different simulation examples. The interfering transmitters use the same OSTBC as the transmitter of interest. The interference-to-noise ratio (INR) is equal to $20 \mathrm{~dB}$ and the QPSK modulation scheme is used. The MIMO channel between the $i$ th transmitter and the receiver is assumed to be quasistatic Rayleigh flat fading with $\left[\boldsymbol{H}_{i}\right]_{n, m} \sim \mathcal{C N}(0,1)$. The channel mismatch $\boldsymbol{\Delta}_{i}$ is assumed to be independent on $\boldsymbol{H}_{i}$ with $\left[\boldsymbol{\Delta}_{i}\right]_{n, m} \sim \mathcal{C N}\left(0, \sigma_{h}^{2}\right)$. We set $\sigma_{h}^{2}=0.1$.

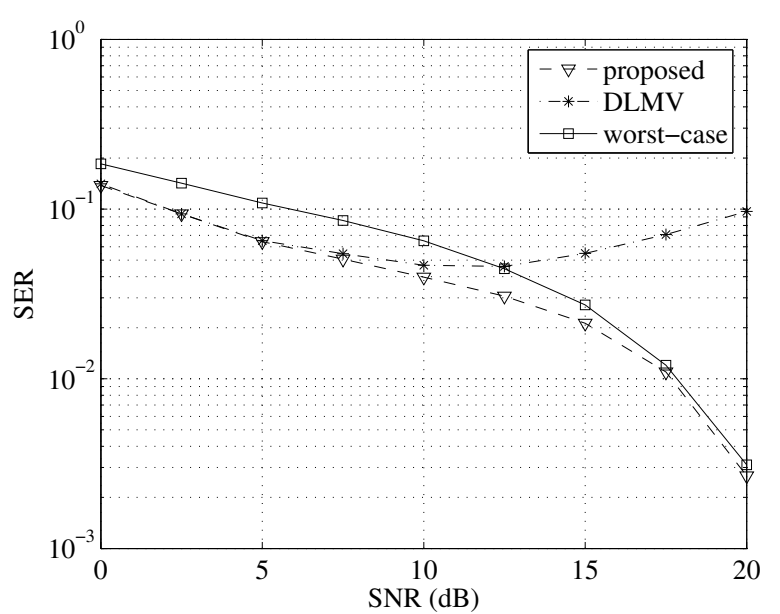

Fig. 2. SER versus SNR; Second example.

The following receivers are compared in terms of symbol error rates (SERs): the proposed SOCP-based receiver (33)-(36), the worst-case-based receiver (see equation (46) in [6]), and the diagonally loaded MV (DLMV) receiver with diagonal loading factors $\nu=10 \sigma_{v}^{2}$, where $\sigma_{v}^{2}$ is the noise variance. The probability $p$ in the proposed robust receivers is set to be equal to 0.99 . For the worst-case-based receiver, $\varepsilon=7 \sigma_{h}$, as suggested in [6]. 300 Monte Carlo runs are used to obtain each point in the simulations.

In our first example, we simulate a scenario with $I=2$ transmitters. Each transmitter uses $N=2$ antennas. The Alamouti code [2] is applied. Fig. 1 compares aforementioned receivers in terms of SER versus SNR for $J=35$.

In the second example, a scenario with $I=4$ transmitters, where each transmitter is equipped with $N=3$ antennas, is simulated. The half rate $(K=4, T=8)$ OSTBC from [3] is used. Fig. 2 shows the SER performance of the aforementioned methods versus SNR when $J$ is equal to 130 .

Figs. 1 and 2 clearly demonstrate that the proposed robust receiver consistently enjoy the best performance as compared with other methods tested. Moreover, the proposed receiver (33)-(36) outperforms the worst-case-based receiver, especially in low and medium SNRs regions.

\section{CONCLUSIONS}

A new approach to design robust linear receivers for multiaccess space-time block coded MIMO wireless communication systems has been proposed. The receivers designed based on this approach provide the robustness against CSI errors with a certain selected probability, which can be determined according to a given QoS. Such receivers can be 
written in terms of stochastic optimization problems with probability constraints. Two such problems were formulated and the connection of one of them to the receiver designed based on the worst-case approach [6] has been shown. Simulation results demonstrate an improved performance of the proposed robust receiver as compared to other known receivers in the presence of CSI errors.

\section{REFERENCES}

[1] A. J. Paulraj and C. B. Papadias, "Space-time processing for wireless communications," IEEE Signal Processing Mag., vol. 14, pp. 49-83, Nov. 1997.

[2] S. M. Alamouti, "A simple transmitter diversity scheme for wireless communications," IEEE J. Select. Areas Commun., vol. 16, pp. 1451-1458, Oct. 1998.

[3] V. Tarokh, H. Jafarkhani, and A. R. Calderbank, "Space-time block codes from orthogonal designs," IEEE Trans. Information Theory, vol. 45, pp. 14561467, July 1999.

[4] H. Li, X. Lu, and G. B. Giannakis, "Capon multiuser receiver for CDMA systems with space-time coding," IEEE Trans. Signal Processing, vol. 50, pp. 11931204, May 2002.

[5] S. Shahbazpanahi, M. Beheshti, A. B. Gershman, M. Gharavi-Alkhansari, and K. M. Wong, "Minimum variance linear receivers for multi-access MIMO wireless systems with space-time block coding," IEEE Trans. Signal Processing, vol. 52, pp. 3306-3313, Dec. 2004.

[6] Y. Rong, S. Shahbazpanahi, and A. B. Gershman, "Robust linear receivers for space-time block coded multiaccess MIMO systems with imperfect channel state information," IEEE Trans. Signal Processing, to appear in Aug. 2005.
[7] S.A. Vorobyov, A.B. Gershman, and Z.-Q. Luo, "Robust adaptive beamforming using worst-case performance optimization: A solution to the signal mismatch problem," IEEE Trans. Signal Processing, vol. 51, No. 2, pp. 313-324, Feb. 2003.

[8] Y. Rong, S. A. Vorobyov, and A. B. Gershman, "A robust linear receiver for multi-access space-time block coded MIMO systems based on probabilityconstrained optimization," Proc. VTC'04 - Spring, Milan, Italy, May 2004, vol. 1, pp. 118-122.

[9] A. Prékopa, Stochastic Programming. Dordrecht, Netherlands: Kluwer Academic Publishers, 1995.

[10] S. Boyd and L. Vandenberghe, Convex Optimization. Cambridge: Cambridge University Press, 2003.

[11] B. Hassibi and B. M. Hochwald, "High-rate codes that are linear in space and time," IEEE Trans. Information Theory, vol. 48, pp. 1804-1824, July 2002.

[12] M. Gharavi-Alkhansari and A. B. Gershman, "Constellation space invariance of orthogonal space-time block codes," IEEE Trans. Information Theory, vol. 51, pp. 331-334, Jan. 2005.

[13] Y. Rong, S. Shahbazpanahi, and A. B. Gershman, "Exploiting the structure of OSTBCs to improve the robustness of worst-case optimization based linear multi-user MIMO receivers," Proc. ICASSP'05, Philadelphia, PA, March 2005, vol. 4, pp. 781-784.

[14] Y. Nesterov and A. Nemirovski, Interior Point Polynomial Algorithms in Convex Programming. Philadelphia, PA: SIAM, 1994.

[15] J. F. Sturm, "Using SeDuMi 1.02, a MATLAB toolbox for optimization over symmetric cones," Optim. Meth. and Software, vol. 11-12, pp. 625-653, Aug. 1999. 